\title{
Distinct repeat motifs at the C-terminal region of CagA of Helicobacter pylori strains isolated from diseased patients and asymptomatic individuals in West Bengal, India
}

Santanu Chattopadhyay ${ }^{1}$, Rajashree Patra ${ }^{1}$, Raghunath Chatterjee ${ }^{1}$, Ronita De ${ }^{1}$, Jawed Alam¹, T. Ramamurthy ${ }^{1}$ Abhijit Chowdhury ${ }^{2}$, G. Balakrish Nair ${ }^{1}$, Douglas E. Berg ${ }^{3}$ and Asish K. Mukhopadhyay ${ }^{1,4^{*}}$

\begin{abstract}
Background: Infection with Helicobacter pylori strains that express CagA is associated with gastritis, peptic ulcer disease, and gastric adenocarcinoma. The biological function of CagA depends on tyrosine phosphorylation by a cellular kinase. The phosphate acceptor tyrosine moiety is present within the EPIYA motif at the C-terminal region of the protein. This region is highly polymorphic due to variations in the number of EPIYA motifs and the polymorphism found in spacer regions among EPIYA motifs. The aim of this study was to analyze the polymorphism at the C-terminal end of CagA and to evaluate its association with the clinical status of the host in West Bengal, India.

Results: Seventy-seven H. pylori strains isolated from patients with various clinical statuses were used to characterize the C-ternimal polymorphic region of CagA. Our analysis showed that there is no correlation between the previously described CagA types and various disease outcomes in Indian context. Further analyses of different CagA structures revealed that the repeat units in the spacer sequences within the EPIYA motifs are actually more discrete than the previously proposed models of CagA variants.

Conclusion: Our analyses suggest that EPIYA motifs as well as the spacer sequence units are present as distinct insertions and deletions, which possibly have arisen from extensive recombination events. Moreover, we have identified several new CagA types, which could not be typed by the existing systems and therefore, we have proposed a new typing system. We hypothesize that a cagA gene encoding higher number EPIYA motifs may perhaps have arisen from cagA genes that encode lesser EPIYA motifs by acquisition of DNA segments through recombination events.
\end{abstract}

Keywords: Helicobacter pylori, CagA, Duodenal ulcer

\section{Background}

The gastric pathogen Helicobacter pylori chronically infect more than half of human population. Although most infections are asymptomatic, $10-15 \%$ of the H. pylori infected individuals develop chronic inflammation leading to atrophic gastritis, peptic ulcer as well as

\footnotetext{
* Correspondence: asish_mukhopadhyay@yahoo.com

'National Institute of Cholera and Enteric Diseases, Kolkata, 700010, India ${ }^{4}$ Division of Bacteriology, National Institute of Cholera and Enteric Diseases, P 33 CIT Road Scheme XM, Beliaghata, Kolkata, 700010, India

Full list of author information is available at the end of the article
}

gastric adenocarcinoma [1,2].This pathogen was classified as a type I carcinogen by the World Health Organization in 1994. However, specific traits that enable a small proportion of this genetically diverse bacterium in the pathogenesis are poorly understood. Early studies showed that human convalescent sera collected from diseased patients significantly respond to a high molecular weight immunodominant bacterial protein known as CagA [3-5]. Subsequently, it was found that in western countries, $H$. pylori strains that express CagA and specific vacuolating cytotoxin (VacA) subtypes are

\section{Biomed Central}

(c) 2012 Chattopadhyay et al.; licensee BioMed Central Ltd. This is an Open Access article distributed under the terms of the Creative Commons Attribution License (http://creativecommons.org/licenses/by/2.0), which permits unrestricted use, distribution, and reproduction in any medium, provided the original work is properly cited. 
significantly associated with the diseases [6-10]. Interestingly, the expression of these virulence markers does not seem to be similarly associated with the disease in Asian context [11]. Although a series of hypotheses were recently proposed the specific reason for the differences in disease outcome and the biological function of the CagA in gastric epithelium remained mostly unexplained.

The gene that encodes CagA is part of a $\sim 40$ kb horizontally acquired DNA segment in the $H$. pylori genome known as cag pathogenecity island (cagPAI) [8]. The cagPAI also contain genes encoding a type IV secretion system, to ensure efficient translocation of the CagA protein into the host epithelium [12-16]. After translocation, CagA multimerizes and undergoes tyrosine phosphorylation by Src family kinases to its tyrosine moiety, which resides in a repeat motif of five amino acids, glutamateproline-isoleusine-tyrosine-alanine (EPIYA), located at the C-terminal region of the protein [17-20]. Phosphorylated CagA binds to the Src homology 2 (SH2) domain of the cellular phosphatase SHP-2, an important component of the cell cycle regulation pathway. Due to the intrinsic membrane tethering property of CagA the CagA-SHP-2 complex localizes to the plasma membrane of the host epithelium [21], leading to disregulation of the SHP-2. This event is necessary and sufficient to change the gastric epithelium to a transformed epithelium, which is characterized by altered cellular proliferation, migration and elongated cell morphology known as hummingbird phenotype [21,22]. The CagA also disrupts the tight junctions and causes loss of apical-basolateral polarity in epithelial cells $[23,24]$.

The extent of biological activity of CagA is directly associated with the number of phosphorylation sites or the number of EPIYA motifs present at the C-terminal region of CagA $[21,25,26]$. Due to these repeats, molecular weight of the CagA protein varies from $128-148 \mathrm{kDa}$ and the $\operatorname{cagA}$ gene shows extensive length polymorphism at the 3' end. Several attempts were made to type the distinct CagA proteins and the $\operatorname{cagA}$ genes on the basis of its length polymorphism at the C-terminal and 3 ' ends, respectively $[9,27-29]$. The simplest method uses a PCR to amplify the 3' end of the gene to distinguish the variations in the number and order of the EPIYA motif coding regions [30]. In this PCR, it was found that the "type A", which gives $~ 642 \mathrm{bp}$ amplicon and encodes three EPIYA repeats is the most prevalent CagA type. This type usually is not associated with the diseases. The "Type C", which yields $~ 810$ bp product, encodes four EPIYA repeats is associated with gastric adenocarcinoma in Japan. Although this nomenclature is useful in terms of assessing disease association of different CagA types, the validity of this typing system in terms of disease association and the prevalence of various CagA types have not been tested in different geographic regions [31,32].
Another typing method, which was used more often in recent studies addressing the biological function of CagA described the distribution of 5 EPIYA motifs as A-B-CC-C in CagA of strain NCTC11637 [33]. Mutational analyses revealed that the first two EPIYA motifs (A and $B$ positions) have little, if any, biological function while the other motifs (-C-C-C positions) are responsible for the CagA phosphorylation and CagA-SHP-2 complex formation, which leads to the hummingbird phenotype [33]. Moreover, the amino acid sequences, which are repeated among the third, fourth and fifth EPIYA motifs $(-\mathrm{C}-\mathrm{C}-\mathrm{C})$ differ among strains isolated from West and East Asia. Accordingly, CagA was typed as WesternCagA-specific sequences (WSS) and East Asian-CagAspecific sequences (ESS) and this difference may account for differences in disease outcome between the two geographical regions.

The present study aimed to address a number of issues. First, we assessed the disease association with $\operatorname{cagA} 3^{\prime}$ end variations in Indian $H$. pylori strains, where the incidence of gastric cancer is less but the incidence of duodenal ulcer is common. Second, the exact repeat units, which form the spacer regions among the EPIYA motifs, were determined to understand the precise mosaic structure of the CagA phosphorylation site repeat units. Finally, we have identified several new CagA types, which could not be typed with the existing nomenclature and this motivated us to propose a new typing system.

\section{Results}

Prevalence of cagA types and clinical outcome

Fifty-eight (31 of 40 duodenal ulcer patients (DU), 17 of 20 healthy volunteers (HV), 10 of 15 non-ulcer dyspeptics (NUD) and one from gastric adenocarcinoma patients (GC) of $77 \mathrm{H}$. pylori strains included in this study yielded a typical $\sim 642 \mathrm{bp}$ amplicon, designated as type A by Yamaoka et al. (1998) (Table 1). PCR using the same primer pair yielded $\sim 756 \mathrm{bp}$ amplicon corresponding to type B/D for 9 strains (4 from DU, 2 from HV and 3 from NUD) and $\sim 810$ bp amplicon corresponding to type $\mathrm{C}$ for 5 strains (3 from DU, 1 from HV and 1 from NUD) (Figure 1). Two strains from DU (I27i and PCR 98i) and one strain from GC (PCR 93ii)

Table 1 Prevalence of cagA subtypes obtained in Kolkata and disease outcome

\begin{tabular}{llll}
\hline cagA types & DU (N=40) & Clinical statusHV (N=20) & NUD (N=15) \\
\hline Type B/D & $31(77.5 \%)$ & $17(85 \%)$ & $10(66.6 \%)$ \\
Type B/D & $4(10 \%)$ & $2(10 \%)$ & $3(20 \%)$ \\
Type C & $3(7.5 \%)$ & $1(5 \%)$ & $1(6.7 \%)$ \\
New types & $2(5 \%)$ & $0(0 \%)$ & $1(6.7 \%)$
\end{tabular}

* Two strains isolated from gastric carcinoma patients were not shown in the table due to less sample size 


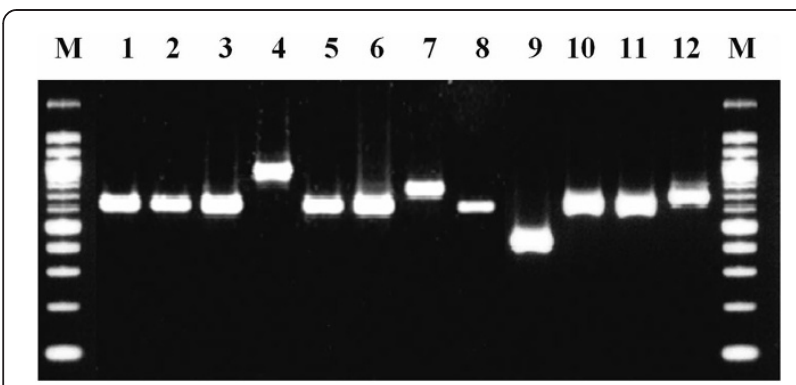

Figure 1 Analysis of the $3^{\prime}$ region of the cagA gene by PCR. PCR products from a representative group of strains are shown. The sizes of the DNA fragments were confirmed after sequencing of the PCR products. Lane M, 100-bp molecular size markers.

gave unusual shorter amplicons ( $550 \mathrm{bp})$. One strain isolated from NUD (PCR 24) yielded the shortest amplicon ( 450 bp) encountered in this study (Table 1). None of these four short amplicons were described in the earlier typing system. No disease association was found with any of the CagA types. The expression of all CagA types was confirmed by Western blot using anti-CagA antidody (Figure 2). [The nucleotide sequences of the 3 ' end of cagA gene of $21 \mathrm{H}$. pylori strains were deposited in GenBank under the following accession numbers: EU089757 to EU089775, EU368669 and EU368670.]

\section{Precise mosaic structure of the CagA}

Nine representative strains with cagA type A (5 from DU and 2 from HV and 1 each from CA and NUD), 6 strains with type B/D cagA (3 each from DU and HV), 2

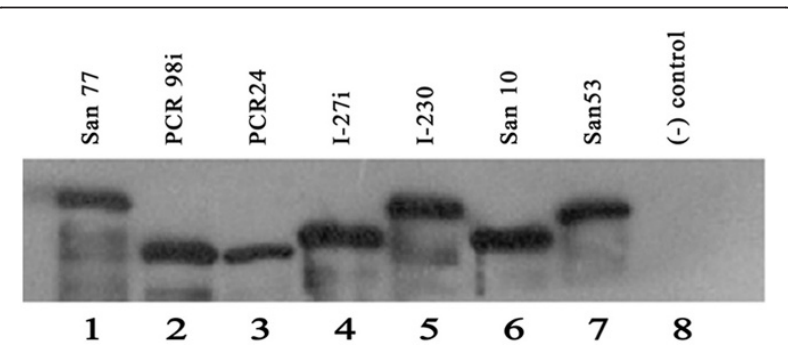

Figure 2 Western blot analysis of $H$. pylori strains expressing representative CagA variants. H. pylori strains were grown on brain heart infusion agar plates, harvested in PBS and subjected to SDS-PAGE followed by western blot analysis using anti-CagA antibody. Strain San 77, which carries CagA with five EPIYA motifs and gave $~ 810 \mathrm{bp}$ amplicon in PCR using primer CAG1/CAG2 expressed CagA protein with highest molecular weight (lane 1). PCR98i and PCR24 which carry only two and single EPIYA motifs, gave $\sim 550$ bp and $\sim 450$ bp amplicon respectively in the same PCR expressed CagA proteins with much smaller size (lane 2 and 3). These two strains expressed CagA proteins, which are smaller than CagA expressed by strain San10 (lane 6) that carries 3 EPIYA motifs and gave 642 bp amplicon, although 1-27i (lane 4), which also carries two EPIYA motifsexpressed CagA protein with higher size than PCR98i (lane 2). Strain 1-80, which carries no cagA gene, was used as negative control (lane 8). strains with cagA type C (1 each from DU and HV) and 4 strains (I-27i, PCR24, PCR93ii and PCR98i) that yielded unusual amplicons were included in the sequence analysis. All the strains used in sequence analysis carried Western-CagA-specific sequences (WSS). These strains carried cagA genes capable of coding proteins containing 1-5 EPIYA motifs. Further analysis of the amino acid sequences predicted from nucleotide sequences of cagA 3 ' end revealed several distinct features present within EPIYA motifs.

To illustrate the discrete repeat units, a CagA protein sequence (San 77) carrying the typical A-B-C-C-C type EPIYA motifs (and WSS), which produced $\sim 810$ bp amplicon with primers CAG1 and CAG2 was taken as model (Figure 3). These short repeat units were detected in CagA sequences of all the strains isolated from West Bengal and Western Countries (those carrying WSS) (Figure 4). The first EPIYA motif (termed as Y1 in this study to denote tyrosine phosphorylation site) in this CagA primary structure was preceded by a 10 amino acids motif (S1) containing a stretch of 6 asparagines (Figure 3). Irrespective of CagA types, this motif was detected in all CagA proteins having WSS (Figure 4).

The first and the second EPIYA (termed as Y2) motifs are separated by a motif named V1 that contains 13 amino acids. The V1 motif was found in all CagA sequences included in this study that carry A-B-C or A$\mathrm{B}-\mathrm{C}-\mathrm{C}$ or $\mathrm{A}-\mathrm{B}-\mathrm{C}-\mathrm{C}-\mathrm{C}$ type EPIYA patterns. In agreement to the previous report, no type D CagA carried similar motif. Our analysis indicated that the absence of V1 and Y2 in type D CagA is due to a specific deletion of this locus (Figure $5 \mathrm{~b}$ ). Detection of several other deletions in several other CagA types further supported this connotation (Figure $5 \mathrm{~b}$ ).

Our analysis also indicates that the spacer regions of third, fourth and fifth EPIYA (Y3, Y4 and Y5) motifs contain several short discrete repeat motifs. The I1 motif was present immediately after the Y2 and was never reappeared (Figure 4). This stretch was followed by I 2 and I3, which was present just before the Y3. As shown in Figures 3 and 4, I2 and I3 were repeated independent of each other but always maintained their respective positions and I2 was repeated one more time than I3.

The $\mathrm{Y} 3$ is followed by another distinct motif, W1, which is followed by $\mathrm{I} 2$ and I3 in A-B-C-C and A-B-C$\mathrm{C}-\mathrm{C}$ type CagA. However, in A-B-C type CagA (Figure 5B, strain PCR29i), though $\mathrm{W} 1$ and $\mathrm{I} 2$ were always present, I3 was replaced by another motif, T1. In A-B-C-C-C type CagA, I3 is repeated 3 times along with EPIYA motifs (at $\mathrm{C}$ positions) and after the last EPIYA at $\mathrm{C}$ position (Y5), I3 was replaced by $\mathrm{T} 1$ (Figure $5 \mathrm{~b}$ ). The comparative analysis of all the CagA primary structures revealed that $\mathrm{T} 1$, which must be present after I2 
(and in the place of I3) indicates the termination of this repeat sequences and after T1 no EPIYA motif was detected in any CagA primary structure.

The differences in PCR product sizes observed between type $\mathrm{C}$ strains isolated from Japan and West Bengal were because the intact $\mathrm{R} 3$ region is repeated twice in ESS carrying CagA reported for Japanese $H$. pylori strains, while CagA found in West Bengal (and also for all WSS carrying CagA), only I2 and I3 is repeated but not I1. Another important feature of WSS carrying CagA is W1

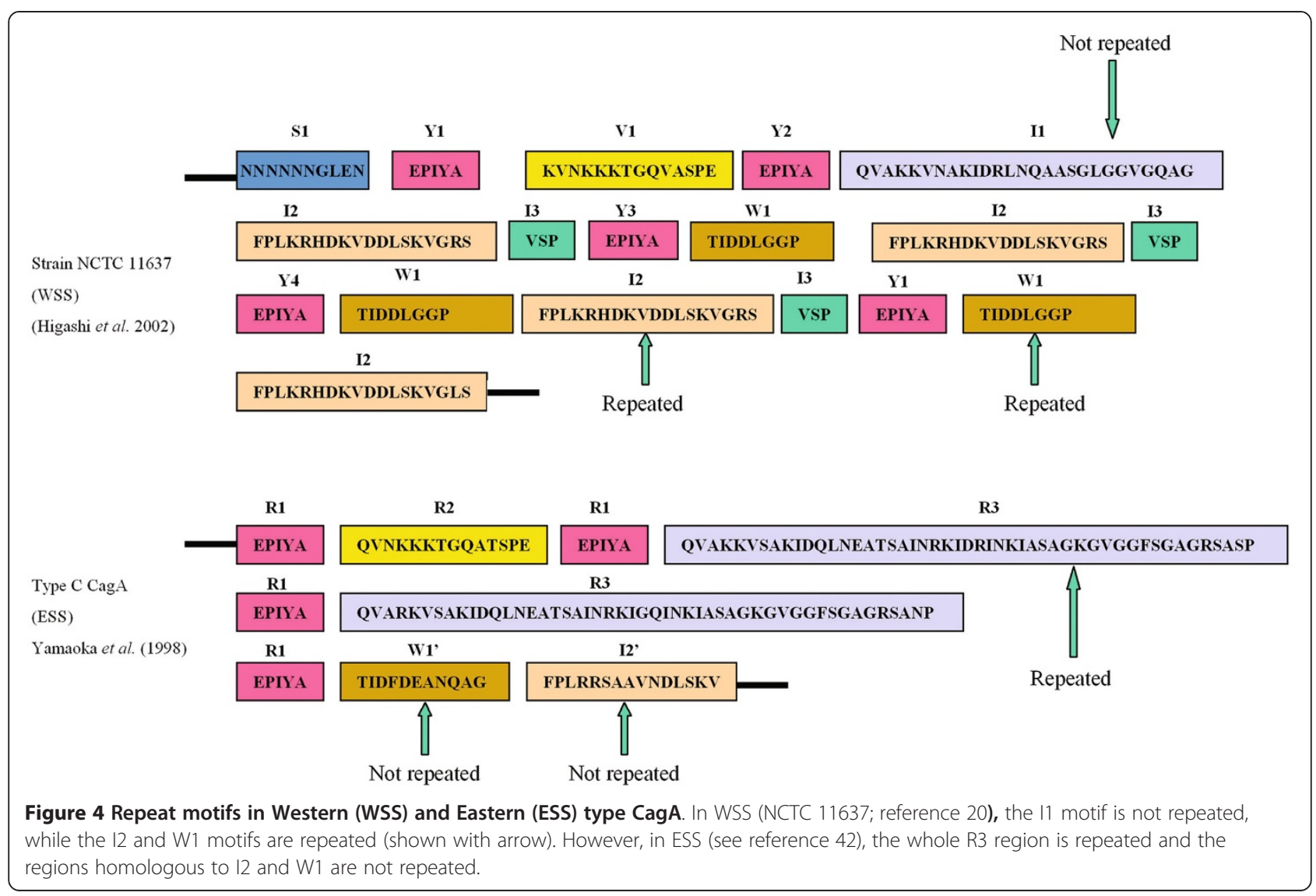




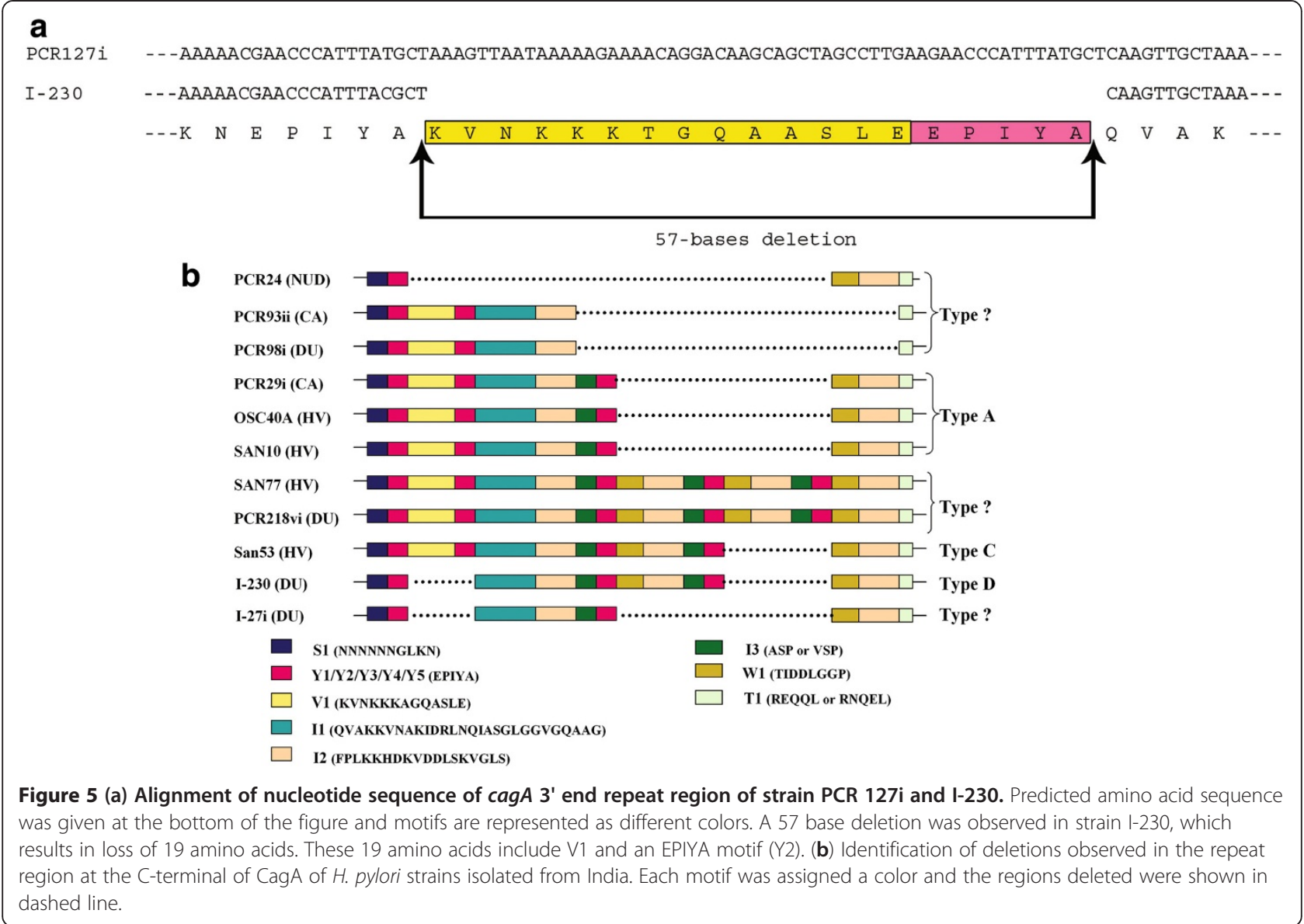

(TIDDLGGP) is repeated after all EPIYA motifs at C positions (Y3, Y4, Y5), while the sequence, which replaces this motif in ESS CagA (TIDFGEANQAG) is present only once, after the last EPIYA motif (Y3 for AB-C type, Y4 for A-B-C-C and Y5 for A-B-C-C-C type). The motif in ESS CagA (FPLRRSAAVRDLSKVGLS), which is homologous to I2, is also present only once, immediately before $\mathrm{T} 1$.

\section{Description of new CagA types and development of new typing system}

Our sequence analyses identified several new CagA primary structures, which could not be typed by previously suggested typing system. The strains that gave unusual $\sim 550$ bp amplicons (PCR93ii and PCR98i) were found to carry unique CagA primary structures. Interestingly, in these two CagA primary structures, I3 and W1 regions were missing. The entire sequence between the $\mathrm{Y} 2$ and the T1 was also absent, possibly due to a deletion event (Figure 5B). In other words, for these two CagA types, EPIYA motifs were present at $A$ and $B$ positions only, while there was no EPIYA motif at $C$ position [33]. However, the strain I-27i, which yielded similar $\sim 550 \mathrm{bp}$ amplicon contains entirely different CagA primary structure. In this CagA, V1 and the adjacent EPIYA (Y2) at $\mathrm{B}$ position were absent (Figure $5 \mathrm{~b}$ ). The shortest amplicon ( $450 \mathrm{bp)}$ was obtained in this study with strain PCR24. In this CagA, the S1 was followed by only one EPIYA motif and then a big deletion was detected as several repeat units such as V1, Y2, I1, I2, I3 and Y3 were entirely missing. Since previous typing systems failed to type these CagA primary structures we suggest a new typing system (Table 2).

\section{Natural insertion and deletions}

Our analyses suggest that the distinct repeat motifs could be present in the form of either deletion or insertion. To further confirm this hypothesis, CagA from 2 strains (San53 and PCR29i) could be taken as examples. As shown in Figure 5, these two primary structures revealed that only a portion of CagA sequence (W1I2I3Y4, shown in underline and italics) in strain San53 was absent in CagA primary structure of strain PCR 29i. In other words, if the region W1I2I3Y4 could be inserted to CagA primary structure of strain PCR 29i, between Y3 and W1 (shown in bold), both these strains would have similar CagA. 
Table 2 CagA primary structures commonly observed in India and in Western countries and the new typing system.

\begin{tabular}{|c|c|c|c|c|}
\hline CagA primary structures & $\begin{array}{l}\text { Types described by } \\
\text { Yamaoka et al. (1998) }\end{array}$ & $\begin{array}{l}\text { Types described by } \\
\text { Higashi et al. (2002) }\end{array}$ & $\begin{array}{l}\text { New typing system } \\
\text { (this study) }\end{array}$ & $\begin{array}{l}\text { Examples in } \\
\text { this study }\end{array}$ \\
\hline S1Y1V1Y2I1I2I3Y3W1I2T1 & Type A & $A-B-C$ & Type 1 & PCR29i \\
\hline S1Y1V1Y2I1|2|3Y3W1|2|3Y4W1|2T1 & Type C & $A-B-C-C$ & Type 2 & San53 \\
\hline S1Y1V1Y2I112I3Y3W1|2|3Y4W1|2|3Y5W1|2T1 & Type? & $A-B-C-C-C$ & Type 3 & San77 \\
\hline S1Y1|1|2I3Y3W1|2|3Y4W1|2T1 & Type D & $A-C-C$ & Type 4 & $1-230$ \\
\hline S1Y1I11213Y3W1|2T1 & Type? & $\mathrm{A}-\mathrm{C}(?)$ & Type 5 & $1-27 i$ \\
\hline S1Y1W1I2T1 & Type ? & $A(?)$ & Type 6 & PCR24 \\
\hline S1Y1V1Y2I1I2T1 & Type? & $A(?)$ & Type 7 & PCR98 \\
\hline
\end{tabular}

Some of these types could not be addressed with previous systems and termed as "type ?"

Analysis also revealed that strains (eg. I-230) carrying previously described type D CagA actually has a deletion of V1Y2 regions and if this region were inserted between Y1 and I1 (shown in bold) they would have a CagA similar to that of type $C$ (Figure 5a). Even more interesting observations were obtained while analyzing CagA primary structure of strain I-27i where region W1I2I3Y4 was deleted. Although this strain gave an amplicon closer to type A by PCR using primers CAG1 and CAG2, primary structure analysis revealed that this CagA was closely related to type D and insertion of W1I2I3Y4 between Y3 and W1 (shown in bold) could make it exactly similar to type D CagA (Figure $5 \mathrm{~b}$ ).

Further larger deletions in CagA were noticed in strains PCR93ii and PCR98i. Both these strains had a deletion of I3Y3W1I2 between I2 and T1 (shown in bold). Strain PCR24, which carry only one EPIYA motif, had the largest deletion. The entire region between $\mathrm{Y} 1$ and W1 (shown in bold) was deleted in this CagA and it was not possible to predict from which type of CagA it has evolved through deletion. On the other hand, it is possible that this CagA bears the core unit and the other complex CagA structures have evolved from it (Figure 6).

\section{Putative recombination breakpoint analysis}

TOPALi is graphical analysis software that uses statistical analysis to detect the presence of possible recombination sequences and gives an approximate location of the breakpoints within DNA multiple alignments [34]. As described in the methods, 3' end sequences of cagA gene of $19 \mathrm{H}$. pylori sequences were aligned, and the putative recombination breakpoints were analyzed by TOPALi (Figure 7). Using TOPALi the recombination breakpoints are predicted at 290, 331, 381 and 447 bp. The sequences between the putative breakpoints 291 and 331 are between Y3 and Y4 motif, and that between 381 and 447 are between Y4 and Y5 motif. The first hypervariable region, predicted using TOPALi, includes Y3, W1 and parts of second I2 motif. The second hypevariable region includes $\mathrm{I} 3, \mathrm{Y} 4, \mathrm{~W} 1, \mathrm{I} 2, \mathrm{I} 3, \mathrm{Y} 5$ and parts of corresponding W1 motif. The predicted recombined regions in the $3^{\prime}$ end sequences of $\operatorname{cag} A$ gene may account for the sequence variation of $\operatorname{cag} A$ gene. This

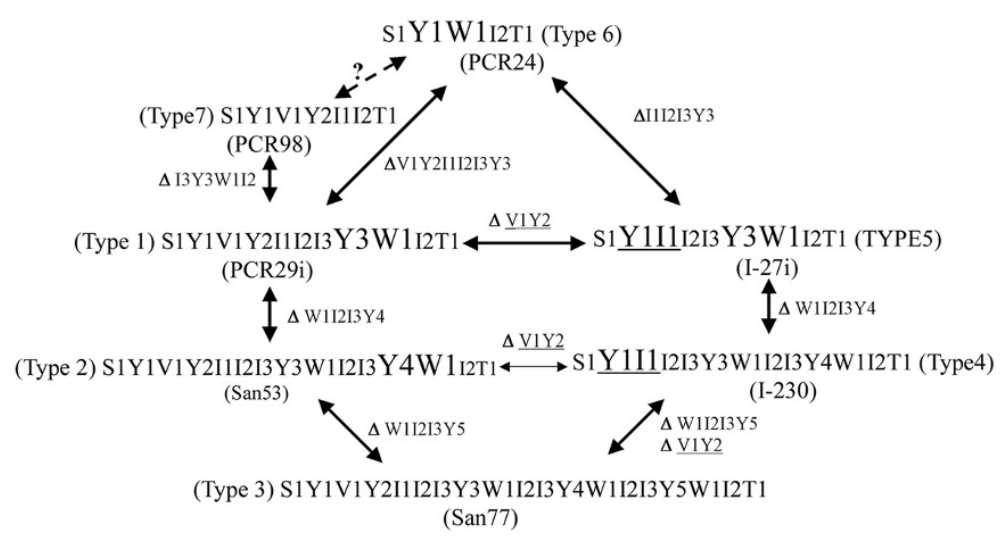

Figure 6 Schematic representation of various types of deletions or insertions among the CagA isolated from WB, India. The segments deleted or inserted are mentioned along with the arrows. The regions where the insertions have occurred are shown in bold and larger front. For type 2 and type 4 CagA, the V1Y2 motifs were inserted within Y1 and 11 and shown in bold, larger front size and also with underscore. As shown in the diagram, almost all the types could be converted to the other types with the occurrence of insertions or deletions. 


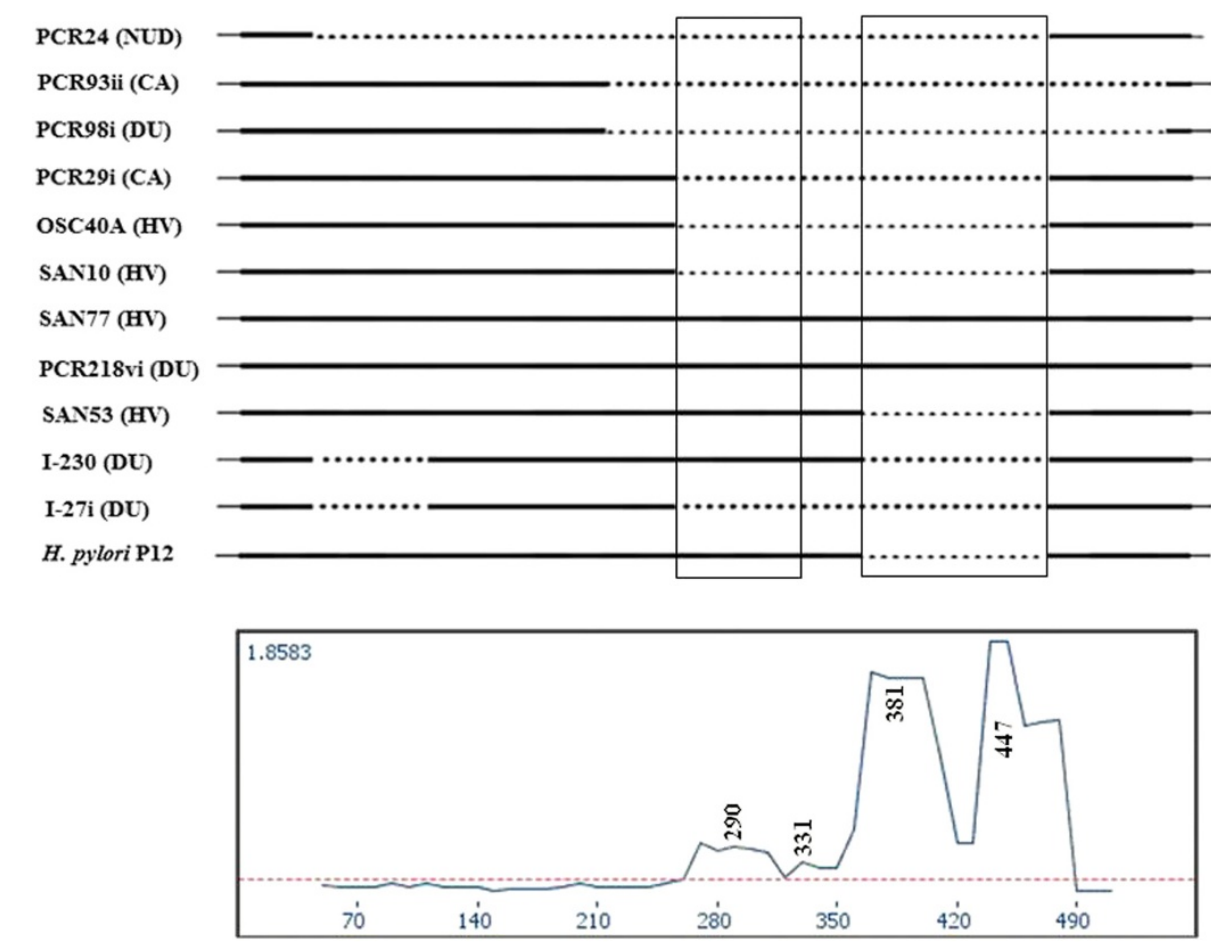

Figure 7 Recombination breakpoint analysis of aligned sequences of $\mathbf{3}^{\prime}$ end of $c a g A$ of $\mathbf{1 1} \mathbf{H}$. pylori strains along with the annotated $\mathrm{H}$. pylori P12 strain using TOPALi. Numbers in bold above and below the peaks indicate the putative recombination breakpoint positions. The dotted horizontal line is the $95 \%$ significance point of Dss. Start of the alignment of $3^{\prime}$ end sequences of cagA corresponds to the 2638 bps from the start of the CagA gene (CP001217) of H. pylori p12 strain.

analysis strengthens the hypothesis that different complex types of $\operatorname{cagA}$ genes are evolved from the CagA that bears the core unit due to the gene conversion at the border of the hypervariable regions.

Disease associations of the $H$. pylori strains are reported to be strongly correlated with the presence of this complex domain architecture with increasing number of repeat sequences containing EPIYA motifs of CagA gene [25,26,30,33]. To assess the possible role of the presence of this motif, we predicted secondary structure of San77, which comprises of the entire five EPIYA motifs (i.e., Y1Y2Y3Y4Y5), using Discrimination of protein Secondary structure Class (DSC) module of Discovery studio (Figure 8). The tyrosine residue in the first and second EPIYA motifs are being contained in the $\alpha$-helical conformation, whereas that of the Y3, Y4 and $\mathrm{Y} 5$ are contained in the beta strand conformation preceding a coil spanning from end of I2 to I3 motif. One may hypothesize that the presence of this tyrosine residue in the $\alpha$-helical conformation may inhibit the phosphorylation of the tyrosine residue by making it less accessible. In contrast, the tyrosine residues in the Y3, Y4 and Y5 motifs might be more exposed and accessible to be phosphorylated due to their presence in $\beta$-strand conformation and the presence of preceding loop conformation. A similar observation was also reported in an earlier study [35]. It could readily explain the reason why CagA proteins having more 34amino acid repeat sequences undergo greater tyrosine phosphorylation and hence exhibit increased SHP-2 binding and induce greater morphological changes.

\section{Discussion}

The length polymorphism observed at the 3 ' end of $\operatorname{cagA}$ gene of $H$. pylori, which results in variation in the number of phosphorylation sites of the encoded protein, is of great interest in recent times since higher number of the phosphorylation sites in CagA was described to be associated with stronger biological function and disease manifestation [25,26,30,33,36]. Several attempts have been made to type this virulence marker considering the variability of the phosphorylation sites (EPIYA) and certain types seem to be associated with the clinical outcome $[9,27-30,33,37,38]$. However, in India, where 85 $90 \%$ of $H$. pylori strains carry the cagA gene [32], data that relate the polymorphism at C-terminal end of the CagA and the clinical status of the host are scanty [39].

We have found that previously described type A (type 1 , in our proposed nomenclature), which is the most prevalent CagA type in other parts of the world, was also detected in West Bengal. However, there was no disease association with any of the previously described CagA 
types. Infection with $H$. pylori strain carrying CagA with higher number of EPIYA motifs (A-B-C-C-C), which was described to be associated with gastric cancer, was also isolated from healthy individuals. The expression of A-B-C-C-C type CagA in H. pylori strain isolated from $\mathrm{HV}$ was also confirmed by western blot analysis. In addition, strains isolated from healthy individuals and those from patients with overt diseases like peptic ulcer or gastric adenocarcinoma showed identical amino acid repeat motifs in the CagA.

Previous reports indicate that the amino acid sequences among the EPIYA motifs vary between Western CagA and East-Asian CagA [25,26,33]. The sequence FPLKRHDKVDDLSKV found in western type CagA (WSS) is replaced by sequence KIASAGKGVGGFSGA in East-Asian type CagA (ESS) and this variation in amino acid sequences might be the reason behind the high prevalence of gastric cancer in Japan as compared to the West $[25,30,33]$. Interestingly, in this study we have found that in Indian context, where the prevalence of duodenal ulcer is more common than gastric cancer, all the strains used for sequence analysis carried western type CagA. This finding is in agreement with previous report which describes that the conserved sequences of cagA 5 ' end region in $H$. pylori strains isolated from Calcutta form cluster with cagA sequences of $H$. pylori strains isolated from western countries and differ significantly from cagA sequences found in East-Asia.

Further analysis of different CagA primary structures revealed that the $\mathrm{C}$-terminal repeat region is actually more discrete from the previously proposed models of CagA variants. For example, from this study it is apparent that the R3 region (originally described by Yamaoka et al for ESS), for WSS CagA, contains 3 distinct repeat regions viz. I1, I2 and I3. The CagA primary structure model proposed by Yamaoka et al. (1998) describes that the $\mathrm{R} 3$ region is present between second and third EPIYA motifs and also between third and fourth EPIYA motifs. However, our analysis with strains isolated from West Bengal and Western countries showed that although intact R3 region (combination of I1-I2-I3) is present between second and third EPIYA motifs ( $\mathrm{Y} 2$ and $\mathrm{Y} 3$ respectively), in type $C$ (type 2 , in the proposed nomenclature) only I2 and I3 is present between third and fourth EPIYA motifs (Y3 and Y4 respectively) and I1 is not repeated. The 34 amino acids repeat region described by Azuma et al. (2004), which is present within third to fifth EPIYA motifs (Y3 to Y5) can be divided as smaller units (Figure 4). The amino acid sequence analysis of CagA in the current study showed that this region includes four distinct, short repeat motifs containing I2, I3, W1 (TIDDLGGP) and Y (EPIYA). For example, in AB-C-C-C type CagA, I2 is repeated four times, while I3 and $\mathrm{W} 1$ repeated three times and after the last EPIYA motif present in position $\mathrm{C}$ (Y5), I3 is replaced by $\mathrm{T} 1$ motif. We have noticed that $\mathrm{T} 1$ motif represents the termination of this repeat region for all CagA types and after this T1 motif, no EPIYA motif was detected.

Our analysis revealed that the EPIYA motifs as well as the spacer sequence units among the EPIYA motifs are present as distinct insertions and deletions. For example, between the first two EPIYA repeats, the identified region named as R2 (V1, in the proposed nomenclature) by Yamaoka et al. (1998) for type C (type 2, in the proposed nomenclature) CagA is absent in type D (type 4, in the proposed nomenclature) CagA, although the remaining motifs are identical. This suggests that the absence of R2 region in type 4 strains is possibly resulted from a deletion. Many other deletions or insertions, which we have shown in our present report, might have occurred due to extensive genetic rearrangements or recombination events during the evolution. In Neisseria gonorrhoeae for example, the pilin genes show extensive variation at the 3 ' end while the $5^{\prime}$ end remain relatively conserved and undergo recombination in order to generate antigenic variation of pilin expression [24]. This reassortment of pilin genes in this organism occurs possibly by two distinct mechanisms [40]. Both piliated and revertible P- cells of $N$. gonorrhoeae are competent for in vitro DNA uptake during autolysis. This mechanism suggests that pilin antigenic variation occurs through transformation by exogenous DNA liberated from lysed cells within a population [41]. In addition to this, transformation-mediated recombination and intragenomic reciprocal recombination also contribute to the pilin antigenic variation [40]. Likewise, H. pylori is also naturally competent [42], undergoes autolysis [43] and the frequency of recombination in this species is very high [44]. This indicates that transformation-mediated recombination might be responsible for the extensive variations observed at the $3^{\prime}$ end of cagA gene. Moreover, the cagPAI contains genes that code for a putative type IV pilus, which is involved in CagA translocation to the host cell could also be involved in the DNA uptake [12,14-16]. Intragenomic reciprocal recombination between two cagA loci of the same chromosome is also possible as multiple $H$. pylori strains were isolated from single gastric niche, which had identical DNA fingerprint with polymorphism only at the 3 ' end repeat region of cagA (unpublished data). It is important to mention here that the CagA, apart from SHP2, interacts with several other proteins, which are important for cell cycle regulation and EPIYA motifs, in phosphorylated as well as unphosphorylated form, are possibly the active sites of CagA $[23,45,46]$. Therefore, recombination in these sites is possibly important for $H$. pylori to manifest different levels of biological activity in order to survive in human gastric mucosa- an environment, which changes often 


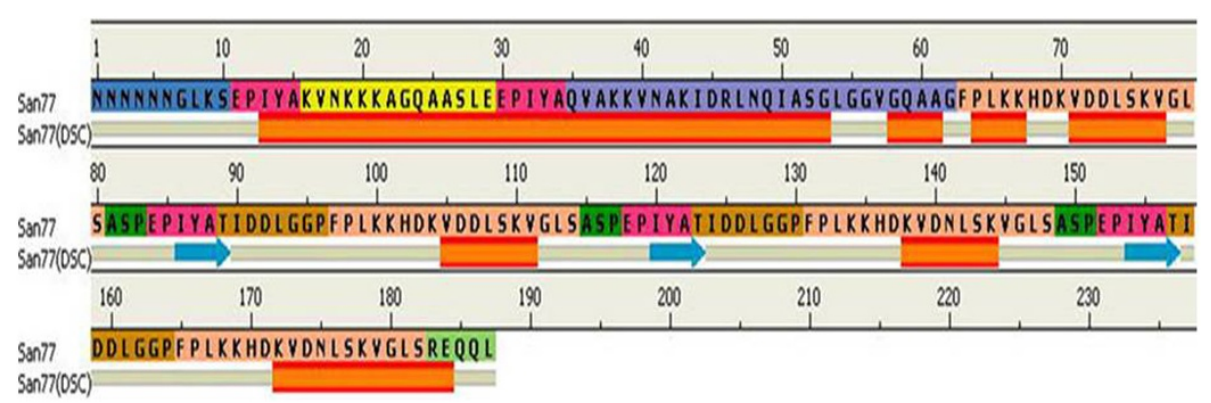

Figure 8 Secondary structure cartoon of San77 predicted using Discrimination of protein Secondary structure Class (DSC) in Discovery studio (Accelrys, USA). Helices are colored in red, strands are in blue, and coils are in beige. Tyrosine residues in first and second EPIYA motifs are within the alpha-helical region. Tyrosine residues in third, fourth and fifth 'EPIYA' motifs are within the beta-strands.

with food habits and other lifestyle of the host. Variation in these repeats should also result in variation in threedimensional structure of CagA protein. These structural changes, apart from the variability in biological function in terms of interfering with several signaling pathways, might result in minute changes in antigenicity, which is possibly necessary for the pathogen to escape host immune surveillance, considering the CagA is a highly immunodominant protein. Therefore, $H$. pylori strains, under continuous selection pressure, may recombine with high efficiency during prolonged colonization in gastric mucosa resulting emergence of different CagA types.

\section{Conclusions}

In Summary, we have identified seven CagA types and several of them are being reported for the first time including a CagA type, which carry a single EPIYA motif. These new CagA structures could not be typed by the existing systems and therefore, we have proposed a new typing system. Although this typing system is developed by analyzing CagA primary structures of $H$. pylori strains isolated in West Bengal, India, we have noticed that it is suitable to type all CagA primary structures having WSS detected in other parts of the world. Previously, it was shown by Higashi et al (2002) that tyrosine phosphorylation at $\mathrm{C}$ position is necessary and sufficient for CagASHP2 complex formation and the tyrosine residues at $\mathrm{A}$ and $\mathrm{B}$ positions have little functional significance. We have identified two strains, which carry CagA with EPIYA motifs present at $\mathrm{A}$ and $\mathrm{B}$ positions ( $\mathrm{Y} 1$ and $\mathrm{Y} 2$ respectively) only but lack the same motif at $C$ position (Y3) as this region is found to be absent due to natural deletion. Interestingly, one of these two strains was isolated from a patient with gastric adenocarcinoma. Moreover, we have found that the H. pylori strain (San 77) isolated from healthy individual can express A-B-C-C-C type CagA. Thus, our data strongly indicate that carrying a particular type of CagA is not the only determinant for the disease outcome especially in the developing countries like India, where multiple infections with different CagA primary structures are possible (unpublished data). We believe that CagA status of the infected $H$. pylori strains as well as other factors must contribute to cause disease and host genetic traits are the most important among them.

\section{Materials and methods}

\section{Patient samples and $H$. pylori strains}

A total of $77 \mathrm{H}$. pylori strains, 40 from DU, 20 from HV, 15 from NUD and 2 from GC were isolated as described earlier [11,32]. In brief, these individuals underwent a non-sedated upper gastrointestinal endoscopy (GIF XQ 30, Olympus Optical Company, Japan) under topical lignocaine anesthesia at the Hospital of the Institute of Post Graduate Medical Education and Research in Kolkata, India. Written informed consent was obtained from each of these individuals as per the recommendations of the Ethical Committee of the Institute of Post Graduate Medical Education and Research and the study was ethically approved by the committee. Gastric biopsy specimens were transported in $1 \mathrm{ml}$ of Brucella broth (Becton Dickinson, Sparks, MD, USA) containing $15 \%$ of glycerol in cold condition for culture at the Helicobacter unit of the National Institute of Cholera and Enteric Diseases. $H$. pylori was isolated from biopsy specimens, sub-cultured and preserved at $-70^{\circ} \mathrm{C}$ in brain heart infusion (BHI) (BD) containing $15 \%$ glycerol as described earlier [11,32].

\section{Characterization of $H$. pylori strains by PCR}

H. pylori genomic DNA was extracted by CTAB (hexadecyltrimethyl ammonium bromide) method [47] from confluent lawn of bacterial culture on BHI agar (BD) plate. All the strains included in this study carried cagA gene as determined previously by PCR using primers designed from $5^{\prime}$ end conserved regions [32,48]. Genomic DNA extracted from these strains was used to 
amplify 3' end repeat region of cagA with primers CAG1 (5'-ACCCTAGTCGGTAATGGGTTA-3') and CAG2 (5'-GTAATTGTCTAGTTTCGC-3') [30].

\section{Nucleotide sequencing of PCR products and sequence analysis}

PCR products were purified by QIAquick PCR purification kit (Qiagen GmbH, Hilden, Germany) and used directly as a template for nucleotide sequencing. Both strands of DNA were sequenced with the Big-dye terminator cycle sequencing kit (Applied Biosystems, Foster city, CA) according to the manufacturer's instructions, using an ABI PRISM 310 sequencer (Applied Biosystems). The nucleotide and deduced amino acid sequences were edited and compared after aligning using SeqMan and EditSeq program of DNASTAR (DNASTAR, Inc). Multiple alignments were done by using ClustalW in MegAlign software (DNASTAR, Inc.). Finally, the discrete motifs present at the $\mathrm{C}$-terminal end of the CagA were identified by manual analysis.

\section{Western blotting}

H. pylori strains cultured on BHI agar were harvested in phosphate-buffered saline (PBS), and subjected to SDSPAGE followed by transfer to nitrocellulose membrane. Proteins immobilized on membrane were incubated with anti-CagA antibody (Austral biologicals, CA) diluted 1:4000 in Tris buffer saline containing $3 \%$ bovine serum albumin and $1 \%$ tween-20 for $1 \mathrm{hr}$, washed and incubated with anti-rabbit antibody (Santa Cruz Biotech., CA) for $1 \mathrm{hr}$. After washing immunoreactive components were visualized by ECL detection system (Amersham).

\section{Nucleotide sequence accession numbers}

The nucleotide sequences of the 3 ' end of cagA gene of $21 \mathrm{H}$. pylori strains were deposited in GenBank under the following accession numbers: EU089757 to EU089775, EU368669 and EU368670.

\section{Sequence and putative recombination breakpoint analysis}

To predict the $\operatorname{cag} A$ recombination sites, the $3^{\prime}$ end sequences of cagA gene of $21 \mathrm{H}$. pylori strains were aligned in ClustalW [49] and some manual corrections were made to achieve accurate alignment. The aligned sequences were analyzed by the TOPALi software. The detailed description of the algorithm of TOPALi software was available elsewhere [34,50,51]. Briefly, the pairwise distance matrix of the aligned sequences was calculated using the Jukes-Cantor nucleotide substitution model. A window $i$ of user-defined length was moved forward and backward along the sequence alignment at a certain stepwise intervals. For each window $i$, the difference of sums of squares of forward $\left(D s s_{F}^{i}\right)$ and backward $\left(D s s_{B}^{i}\right)$ windows were calculated. The corresponding Dss value was obtained using from the maximum of $D s s_{F}^{i}$ and $D s s_{B}^{i}$. Dss values were plotted for each window. A set of adjacent large Dss values forming a significant peak suggested a putative recombination breakpoint.

\section{Competing interests}

The authors declare that they have no competing interests.

\section{Acknowledgement}

The work was supported in part by the Indian Council of Medical Research and Japan Initiative for Global Research Network on Infectious Diseases (JGRID) Ministry of Education, Culture, Sports, Science and Technology of Japan.

\section{Author details}

${ }^{1}$ National Institute of Cholera and Enteric Diseases, Kolkata, 700010, India. ${ }^{2}$ Centre for Liver Research School of Digestive and Liver Diseases, Institute of Post Graduate Medical Education \& Research, Kolkata, India. ${ }^{3}$ Washington University School of Medicine, Saint Louis, USA. ${ }^{4}$ Division of Bacteriology, National Institute of Cholera and Enteric Diseases, P 33 CIT Road Scheme XM, Beliaghata, Kolkata, 700010, India.

\section{Authors' contributions}

SC and AKM conceived of the study, GBN, TR and DEB participated in the design of this study; AC, SC and RP coordinated collection of specimens, maintenance of clinical data and management of patients; SC, RP, RC, RD and JA carried out bacterial isolation and bacterial sequencing and sequence analysis using different soft wares. All authors read and approved the final manuscript.

Received: 4 April 2012 Accepted: 25 May 2012

Published: 25 May 2012

\section{References}

1. Blaser MJ: Helicobacter pylori and the pathogenesis of gastroduodenal inflammation. J Infect Dis 1990, 161:626-633.

2. Peek RM Jr, Blaser MJ: Helicobacter pylori and gastrointestinal tract adenocarcinomas. Nat Rev Cancer 2002, 2:28-37.

3. Cover TL, Dooley CP, Blaser MJ: Characterization of and human serologic response to proteins in Helicobacter pylori broth culture supernatants with vacuolizing cytotoxin activity. Infect Immun 1990, 58:603-610.

4. Cover TL, Glupczynski Y, Lage AP, Burette A, Tummuru MK, Perez-Perez Gl, Blaser MJ: Serologic detection of infection with cagA + Helicobacter pylori strains. J Clin Microbiol 1995, 33:1496-1500.

5. Gerstenecker B, Eschweiler B, Vogele H, Koch HK, Hellerich U, Kist M: Serodiagnosis of Helicobacter pylori infections with an enzyme immunoassay using the chromatographically purified 120 kilodalton protein. Eur J Clin Microbiol Infect Dis 1992, 11:595-601.

6. Atherton JC, Cao P, Peek RM Jr, Tummuru MK, Blaser MJ, Cover TL: Mosaicism in vacuolating cytotoxin alleles of Helicobacter pylori. Association of specific vacA types with cytotoxin production and peptic ulceration. J Biol Chem 1995, 270:17771-17777.

7. Blaser MJ, Perez-Perez Gl, Kleanthous H, Cover TL, Peek RM, Chyou PH, Stemmermann GN, Nomura A: Infection with Helicobacter pylori strains possessing cagA is associated with an increased risk of developing adenocarcinoma of the stomach. Cancer Res 1995, 55:2111-2115

8. Censini S, Lange C, Xiang Z, Crabtree JE, Ghiara P, Borodovsky M, Rappuoli R, Covacci A: cag, a pathogenicity island of Helicobacter pylori, encodes type I-specific and disease-associated virulence factors. Proc Natl Acad Sci U S A 1996, 93:14648-14653.

9. Covacci A, Censini S, Bugnoli M, Petracca R, Burroni D, Macchia G, Massone A, Papini E, Xiang Z, Figura N, et al: Molecular characterization of the 128-kDa immunodominant antigen of Helicobacter pylori associated with cytotoxicity and duodenal ulcer. Proc Natl Acad Sci U S A 1993, 90:5791-5795 
10. Parsonnet J, Friedman GD, Orentreich N, Vogelman H: Risk for gastric cancer in people with CagA positive or CagA negative Helicobacter pylori infection. Gut 1997, 40:297-301.

11. Chattopadhyay S, Datta S, Chowdhury A, Chowdhury S, Mukhopadhyay AK, Rajendran K, Bhattacharya SK, Berg DE, Nair GB: Virulence genes in Helicobacter pylori strains from West Bengal residents with overt $\mathrm{H}$. pylori-associated disease and healthy volunteers. J Clin Microbiol 2002, 40:2622-2625

12. Backert S, Ziska E, Brinkmann V, Zimny-Arndt U, Fauconnier A, Jungblut PR, Naumann M, Meyer TF: Translocation of the Helicobacter pylori CagA protein in gastric epithelial cells by a type IV secretion apparatus. Cell Microbiol 2000, 2:155-164.

13. Odenbreit S, Puls J, Sedlmaier B, Gerland E, Fischer W, Haas R: Translocation of Helicobacter pylori CagA into gastric epithelial cells by type IV secretion. Science 2000, 287:1497-1500.

14. Rohde M, Puls J, Buhrdorf R, Fischer W, Haas R: A novel sheathed surface organelle of the Helicobacter pylori cag type IV secretion system. Mol Microbiol 2003, 49:219-234.

15. Tanaka J, Suzuki T, Mimuro H, Sasakawa C: Structural definition on the surface of Helicobacter pylori type IV secretion apparatus. Cell Microbiol 2003, 5:395-404.

16. Yeo HJ, Savvides SN, Herr AB, Lanka E, Waksman G: Crystal structure of the hexameric traffic ATPase of the Helicobacter pylori type IV secretion system. Mol Cell 2000, 6:1461-1472.

17. Ren S, Higashi H, Lu H, Azuma T, Hatakeyama M: Structural basis and functional consequence of Helicobacter pylori CagA multimerization in cells. J Biol Chem 2006, 281:32344-32352.

18. Selbach M, Moese S, Hauck CR, Meyer TF, Backert S: Src is the kinase of the Helicobacter pylori CagA protein in vitro and in vivo. J Biol Chem 2002, 277:6775-6778.

19. Stein M, Bagnoli F, Halenbeck R, Rappuoli R, Fantl WJ, Covacci A: c-Src/Lyn kinases activate Helicobacter pylori CagA through tyrosine phosphorylation of the EPIYA motifs. Mol Microbiol 2002, 43:971-980.

20. Stein M, Rappuoli R, Covacci A: Tyrosine phosphorylation of the Helicobacter pylori CagA antigen after cag-driven host cell translocation. Proc Natl Acad Sci U S A 2000, 97:1263-1268.

21. Higashi H, Tsutsumi R, Muto S, Sugiyama T, Azuma T, Asaka M, Hatakeyama M: SHP-2 tyrosine phosphatase as an intracellular target of Helicobacter pylori CagA protein. Science 2002, 295:683-686.

22. Yamazaki S, Yamakawa A, Ito Y, Ohtani M, Higashi H, Hatakeyama M, Azuma $\mathrm{T}$ : The CagA protein of Helicobacter pylori is translocated into epithelial cells and binds to SHP-2 in human gastric mucosa. J Infect Dis 2003, 187:334-337.

23. Amieva MR, Vogelmann R, Covacci A, Tompkins LS, Nelson WJ, Falkow S: Disruption of the epithelial apical-junctional complex by Helicobacter pylori CagA. Science 2003, 300:1430-1434.

24. Saunders JR: Bacterial pathogenicity: molecular auspects. In The encyclopedia of molecular biology. Edited by Kendrew J. Oxford, Great Britain: Blackwell science; 1994:101-104.

25. Azuma T: Helicobacter pylori CagA protein variation associated with gastric cancer in Asia. J Gastroenterol 2004, 39:97-103.

26. Azuma T, Yamazaki S, Yamakawa A, Ohtani M, Muramatsu A, Suto H, Ito $Y$, Dojo M, Yamazaki Y, Kuriyama M, et al: Association between diversity in the Src homology 2 domain-containing tyrosine phosphatase binding site of Helicobacter pylori CagA protein and gastric atrophy and cancer. $\mathrm{J}$ Infect Dis 2004, 189:820-827.

27. Argent $\mathrm{RH}$, Zhang $\mathrm{Y}$, Atherton JC: Simple method for determination of the number of Helicobacter pylori CagA variable-region EPIYA tyrosine phosphorylation motifs by PCR. J Clin Microbiol 2005 43:791-795

28. Dong Q, O'Sullivan M, Hall W, Herra C, Kean C, O'Morain C, Buckley $M$ : Identification of a new segment involved in $\operatorname{cag} A 3^{\prime}$ region variation of Helicobacter pylori. FEMS Immunol Med Microbiol 2002, 33:51-55.

29. Owen RJ, Sharp SI, Chisholm SA, Rijpkema S: Identification of cagA tyrosine phosphorylation DNA motifs in Helicobacter pylori isolates from peptic ulcer patients by novel PCR-restriction fragment length polymorphism and real-time fluorescence PCR assays. J Clin Microbiol 2003, 41:3112-3118.

30. Yamaoka Y, Kodama T, Kashima K, Graham DY, Sepulveda AR: Variants of the 3 ' region of the cagA gene in Helicobacter pylori isolates from patients with different $H$. pylori-associated diseases. J Clin Microbiol 1998, 36:2258-2263.

31. Rota CA, Pereira-Lima JC, Blaya C, Nardi NB: Consensus and variable region PCR analysis of Helicobacter pylori 3' region of cagA gene in isolates from individuals with or without peptic ulcer. J Clin Microbiol 2001, 39:606-612.

32. Mukhopadhyay AK, Kersulyte D, Jeong JY, Datta S, Ito Y, Chowdhury A, Chowdhury S, Santra A, Bhattacharya SK, Azuma T, et al: Distinctiveness of genotypes of Helicobacter pylori in Calcutta, India. J Bacteriol 2000, 182:3219-3227.

33. Higashi H, Tsutsumi R, Fujita A, Yamazaki S, Asaka M, Azuma T, Hatakeyama M: Biological activity of the Helicobacter pylori virulence factor CagA is determined by variation in the tyrosine phosphorylation sites. Proc Nat/ Acad Sci U S A 2002, 99:14428-14433.

34. McGuire G, Wright F, Prentice MJ: A graphical method for detecting recombination in phylogenetic data sets. Mol Biol Evol 1997, 14:11251131.

35. Ma Y, Cunningham ME, Wang X, Ghosh I, Regan L, Longley BJ: Inhibition of Spontaneous Receptor Phosphorylation by Residues in a Putative $\propto-$ Helix in the KIT Intracellular Juxtamembrane Region. J Biol Chem 1999, 274:13399-13402.

36. Zhang Y, Argent RH, Letley DP, Thomas RJ, Atherton JC: Tyrosine phosphorylation of CagA from Chinese Helicobacter pylori isolates in AGS gastric epithelial cells. J Clin Microbiol 2005, 43:786-790.

37. Argent RH, Kidd M, Owen RJ, Thomas RJ, Limb MC, Atherton JC: Determinants and consequences of different levels of CagA phosphorylation for clinical isolates of Helicobacter pylori. Gastroenterology 2004, 127:514-523.

38. Yamaoka Y, El-Zimaity HM, Gutierrez O, Figura N, Kim JG, Kodama T, Kashima K, Graham DY: Relationship between the $\operatorname{cagA} 3$ ' repeat region of Helicobacter pylori, gastric histology, and susceptibility to low $\mathrm{pH}$. Gastroenterology 1999, 117:342-349.

39. Tiwari SK, Sharma V, Sharma VK, Gopi M, Saikant R, Nandan A, Bardia A Gunisetty S, Katikala P, Habeeb MA, Khan AA, Habibullah CM: Phylogenetic analysis, based on EPIYA repeats in the cagA gene of Indian Helicobacter pylori, and the implications of sequence variation in tyrosine phosphorylation motifs on determining the clinical outcome. Genet $\mathrm{Mol}$ Biol 2011, 34:280-285.

40. Gibbs CP, Reimann BY, Schultz E, Kaufmann A, Haas R, Meyer TF: Reassortment of pilin genes in Neisseria gonorrhoeae occurs by two distinct mechanisms. Nature 1989, 338:651-652.

41. Seifert HS, Ajioka RS, Marchal C, Sparling PF, So M: DNA transformation leads to pilin antigenic variation in Neisseria gonorrhoeae. Nature 1988 336:392-395.

42. Nedenskov-Sorensen P, Bukholm G, Bovre K: Natural competence for genetic transformation in Campylobacter pylori. J Infect Dis 1990, 161:365-366.

43. Marsich E, Zuccato P, Rizzi S, Vetere A, Tonin E, Paoletti S: Helicobacter pylori expresses an autolytic enzyme: gene identification, cloning, and theoretical protein structure. J Bacteriol 2002, 184:6270-6279.

44. Suerbaum S, Smith JM, Bapumia K, Morelli G, Smith NH, Kunstmann E, Dyrek I, Achtman M: Free recombination within Helicobacter pylori. Proc Natl Acad Sci U S A 1998, 95:12619-12624.

45. Mimuro H, Suzuki T, Tanaka J, Asahi M, Haas R, Sasakawa C: Grb2 is a key mediator of Helicobacter pylori CagA protein activities. Mol Cell 2002, 10:745-755.

46. Saadat I, Higashi H, Obuse C, Umeda M, Murata-Kamiya N, Saito Y, Lu H, Ohnishi N, Azuma T, Suzuki A, Ohno S, Hatakeyama M: Helicobacter pylori CagA targets PAR1/MARK kinase to disrupt epithelial cell polarity. Nature 2007, 447:330-333

47. Ausubel FM, Brent R, Kingston RE, Moore DD, Seidman JG, et al: Current protocols in molecular biology. New York: Greene Publishing and WileyInterscience; 1993.

48. Chattopadhyay S, Patra R, Ramamurthy T, Chowdhury A, Santra A, Dhali GK, Bhattacharya SK, Berg DE, Nair GB, Mukhopadhyay AK: Multiplex PCR assay for rapid detection and genotyping of Helicobacter pylori directly from biopsy specimens. J Clin Microbiol 2004, 42:2821-2824.

49. Thompson JD, Higgins DG, Gibson TJ: CLUSTAL W: improving the sensitivity of progressive multiple sequence alignment through sequence weighting, position-specific gap penalties and weight matrix choice. Nucleic Acids Res 1994, 22:4673-4680. 
50. McGuire G, Wright F: TOPAL: recombination detection in DNA and protein sequences. Bioinformatics 1998, 14:219-220.

51. McGuire G, Wright F: TOPAL 2.0: improved detection of mosaic sequences within multiple alignments. Bioinformatics 2000 16:130-134

doi:10.1186/1757-4749-4-4

Cite this article as: Chattopadhyay et al:: Distinct repeat motifs at the C-terminal region of CagA of Helicobacter pylori strains isolated from diseased patients and asymptomatic individuals in West Bengal, India. Gut Pathogens 2012 4:4.

\section{Submit your next manuscript to BioMed Central and take full advantage of:}

- Convenient online submission

- Thorough peer review

- No space constraints or color figure charges

- Immediate publication on acceptance

- Inclusion in PubMed, CAS, Scopus and Google Scholar

- Research which is freely available for redistribution 